\title{
TRADUCCIÓN Y LEXICOGRAFÍA: UN DIÁLOGO NECESARIO
}

\author{
Cesáreo Calvo Rigual \\ Cesareo.Calvo@uv.es \\ Universitat de València - IULMA \\ Maria Vittoria Calvi \\ maria.calvi@unimi.it \\ Università degli Studi di Milano
}

\section{Resumen}

Se comparan en primer lugar los campos de la Traducción y de la Lexicografía, poniendo de relieve las coincidencias y diferencias de tipo general que hay entre ambas disciplinas. A continuación se hace un repaso de la bibliografía (no demasiado extensa) sobre el tema, destacando que la mayoría de los trabajos se han centrado en el uso de los diccionarios por parte de los traductores, su utilidad, qué tipo de diccionarios prefieren estos y otros aspectos relacionados. Se pone de relieve el escaso interés de los lexicógrafos por el campo de la traducción, al menos como posible fuente para la redacción de diccionarios. Se dejan abiertos varios caminos que podrían ser recorridos con provecho mutuo por traductores y lexicógrafos.

\begin{abstract}
Firstly, the fields of Translation and Lexicography are compared and their similarities and differences are brought to the fore. Later, a review of the (slightly scarce) literature on the topic shows that most of the work has focused on how translators use dictionaries, how useful they are, and which kinds of dictionaries are preferred, among other similar issues. It is highlighted that lexicographers show little interest in the field of translation, at least as a source for dictionary building. A few potential avenues of research are defined, which could be beneficial for translators and lexicographers alike.
\end{abstract}

Palabras clave: Traducción. Lexicografía. Diccionario. Diccionario bilingüe.

Keywords: Translation. Lexicography. Dictionary. Bilingual dictionary. 

The bilingual dictionary is the translator's single, first and most important aid, and a translator who does not consult one when in doubt is arrogant or ignorant or both.

(Newmark 1998: 29)

\section{Introducción. Cuestiones generales}

Traducción y Lexicografía ${ }^{1}$ son dos disciplinas que tienen evidentemente mucho en común y que pueden hacer mucho la una por la otra. Sin embargo, esta relación no es ni ha sido todo lo fluida que cabría desear. Krista Varantola (1998: 180) describe así esta insatisfactoria situación:

the most sophisticated dictionary users are also the most demanding, more suspicious and harder to please than linguistically less sophisticated users who often have reasonably straightforward problems to solve. Consequently, frustration will drive language professionals to denounce dictionaries as inadequate. The situation is polarised, because dictionary makers, equally frustrated, believe that their critics do not understand the effect that space constraints have on the amount and type of information that dictionaries can provide, and are moreover convinced that, as few users read the introductory matter, they have unrealistic expectations about the coverage of their dictionaries.

De manera similar hace oír su voz Reinhard R. K. Hartmann (1989a: 18):

I would appeal to you to increase your awareness of the channels of communication which are there but sometimes unused. Translators, translation theorists, dictionary makers and metalexicographers in German-speaking countries don't read the publications of their French colleagues and vice versa, English and American experts in these fields don't read either. Translators ignore lexicographers, monolingual lexicographers ignore the work of their bilingual colleagues, the people working in so-called general areas ignore those in so-called technical specialisms. We can only function efficiently in society if we keep our own houses in order.

1. A lo largo de este trabajo utilizaremos algunas siglas para evitar la repetición de algunas palabras frecuentes: $\mathrm{T}=$ traducción, traductología; $\mathrm{L}=$ lexicografía; $\mathrm{D}(\mathrm{DD})=$ diccionario (diccionarios); DB (DDBB) = diccionario bilingüe (diccionarios bilingües); DM $(\mathrm{DDMM})=$ diccionario monolingüe (diccionarios monolingües). 
Otros autores (como Roberts 1992: 49 o Rogers y Khurshid 1998: 193), consideran la relación entre traductor y diccionario como una relación de amor-odio. Marello (1989: 119-120) señala que en el momento de usar el diccionario se producen situaciones de idilio entre el traductor y sus DD (cuando encuentra el equivalente adecuado, que incluso no conocía), junto con momentos de "calma operosa" (cuando el D ofrece equivalentes que no son malos, pero resultan inadecuados al contexto) y otros tormentosos (cuando los equivalentes son claramente erróneos o cuando la palabra no se encuentra).

Para intentar paliar esta insatisfacción, en este artículo evaluaremos el estado de la cuestión, examinando los estudios que hasta nuestros días han puesto en relación $\mathrm{T}$ y $\mathrm{L}$, destacando en particular los avances producidos y los posibles desarrollos en el futuro.

Como hemos afirmado al principio, $\mathrm{T}$ y $\mathrm{L}$ son dos disciplinas que tienen mucho en común, aunque presentan también algunas diferencias significativas:

1. Ambas son actividades humanas que cuentan con una antigüedad respetable. La traducción oral fue sin duda practicada desde tiempo inmemorial y las primeras traducciones escritas aparecen poco después de la invención de la escritura, hacia el 2000 a.C. (Van Hoof 1991: 7). Más o menos por la misma época se redactan en la antigua Mesopotamia listas bilingües de palabras, antecedentes de los DDBB (Marello 1989: 8). Tanto en un caso como en otro es evidente su finalidad: facilitar la comprensión entre pueblos que hablaban lenguas diferentes y mutuamente ininteligibles.

2. Frente a esta precocidad, deberemos esperar hasta la segunda mitad del siglo XX para hallar las primeras teorizaciones de ambas disciplinas. En efecto, en el caso de la T, si bien encontramos ciertas reflexiones sobre ella desde muy antiguo, será en los años 50 de dicho siglo cuando aparezcan las primeras obras de un cierto empaque, aunque realmente será en los años 60 y 70 cuando se publiquen las primeras teorías dignas de tal nombre, en su mayoría ligadas aún estrechamente a la Lingüística: Darbelnet y Vinay, Catford, Nida, etc. (Hurtado 2001: 123 ss.). Tras ellas $-y$ hasta nuestros días- hemos asistido a una multiplicación de los enfoques teóricos en T. También la L hubo de esperar hasta época reciente para contar con una teoría orgánica. Si dejamos a un lado la tentativa temprana aunque aislada del ruso Lev V. Scerba en los primeros años 40 del siglo XX, vemos que desde entonces ha habido solo otras cuatro (según Tarp 2008: 14 ss.): las de Franz J. Haussman y Herbert E. Wiegand en los 70, la de Hans-P. 
Kromann y otros autores en los 80 y la teoría funcional de Henning Bergenholtz y otros autores en los 90 . Hay, pues, una diferencia notable en cuanto al número de reflexiones teóricas a las que ambas han dado lugar: la $\mathrm{T}$ cuenta con una amplia gama de teorías (que responden a presupuestos y orientaciones muy variados), mientras que la $\mathrm{L}$ posee un número mucho más limitado, fruto quizá de una necesidad menos sentida de contar con un aparato teórico, todo ello a pesar de que tanto en un caso como en otro prima la práctica sobre la teoría. En este sentido cabe señalar que muchos de quienes se dedican profesionalmente a traducir y a elaborar DD ignoran o incluso niegan la necesidad de un aparato teórico o la utilidad de los avances teóricos que se producen.

3. La T y la L inician su camino hacia la consolidación teórica desde una situación de dependencia de la Lingüística, como subdisciplinas de la Lingüística Aplicada, hasta alcanzar un momento de madurez en el que se reivindican como disciplinas o ciencias independientes. Ese momento llega en ambos casos casi al unísono. Se considera que la primera formulación de la $\mathrm{T}$ en ese sentido es el fundamental trabajo de James S. Holmes titulado "The Name and Nature of Translation Studies", de 1972. En el caso de la L se trata de otra comunicación presentada en un congreso de la disciplina por parte de H. E. Wiegand en 1977 ("On the structure and contents of a general theory of lexicography").

4. El grado de desarrollo alcanzado es muy diferente. Basta examinar algunos de los indicadores siguientes:

- El estatus académico: existen hoy en todo el mundo numerosas escuelas y facultades de traducción e infinidad de titulaciones en todos los niveles universitarios en traducción e interpretación o en materias afines; son prácticamente inexistentes en el campo de la L, con algunas excepciones como los centros de Lexicografía de Augsburgo, de Aarhus o de Exeter, además de algunos másteres o estudios equivalentes (no existen, según mis noticias, titulaciones de primer grado en $\mathrm{L}$ ).

- Las revistas científicas: las primeras en el ámbito de la T, que siguen publicándose hoy en día (Traduire, Babel, Meta) echan a andar a mediados de los 50 del siglo XX. Las dos revistas más relevantes del campo de la L son más recientes: Lexicographica (1985) e International Journal of Lexicography (1987). En la actualidad existe un número de revistas de T muy superior al de las de L. 
- Publicaciones monográficas, congresos: también aquí el balance es netamente positivo en cuanto a su cantidad para la $\mathrm{T}$.

- Asociaciones: a título de ejemplo, en España se cuentan no menos de 15 asociaciones de traductores (e intérpretes), que tienen todas ellas un carácter profesional y solo en algún caso tangencialmente académico; frente a ellas, en el campo lexicográfico existe una sola, a la que pertenecen lexicógrafos y, sobre todo, estudiosos de la L.

5. El concepto de equivalencia (de traducción) es esencial en $T$ y en la L bilingüe o multilingüe, aunque existe una diferencia esencial: mientras la equivalencia en $\mathrm{T}$ es fundamentalmente contextual, en $\mathrm{L}$ suele hacerse abstracción del contexto, o, dicho de otro modo, se hace abstracción del máximo número de contextos de una unidad léxica para ofrecer equivalentes que sirvan en los contextos más habituales de dicha unidad. Sobre este tema, tanto en $\mathrm{T}$ como en $\mathrm{L}$, han corrido ríos de tinta: valga como ejemplo el tratamiento que del concepto en ambas disciplinas hace Hartmann (1989a).

Así pues -y a pesar de las diferencias- no hay duda de que se trata de dos ámbitos que tienen mucho en común y que, además, en nuestra opinión, pueden aportar mucho el uno al otro. Vamos a comprobar a continuación, mediante la evaluación de la bibliografía existente, si existe un interés real mutuo.

Las obras de referencia de T y L suelen ignorar estas relaciones, ${ }^{2}$ aunque es cierto que en los dos trabajos fundacionales citados antes (Holmes, Wiegand) se delimitan con precisión los subcampos en los que pueden desarrollarse sus estudios y en los dos aparece la disciplina hermana. Holmes (cit. en Hurtado 2001: 138) distingue entre estudios puros y aplicados en T; estos últimos comprenden tres campos: la didáctica de la $\mathrm{T}$, la crítica de traducciones y las herramientas de $T$, entre las que se encuentran dos tipos de obras, los DD y las gramáticas; sin embargo, Holmes considera este tercer campo como

2. Para no recargar en exceso la bibliografía final, nos limitaremos a citar los nombres de algunos de los principales especialistas en ambos campos, que resultan bien conocidos para los cultivadores de las dos disciplinas. Como hemos dicho, todos ellos ignoran generalmente el otro. En el campo de la T, desde los estudios clásicos de J. C. Catford, Nida y Taber o Vázquez Ayora, hasta los más recientes de M. Baker, Hatim y Mason, C. Nord, etc. Como veremos más adelante, encontraremos algunas excepciones entre quienes se han interesado por ambos campos, como Hartmann y Snell-Hornby, o en ciertos autores cuyas teorías están sólidamente unidas a su propia práctica traductora, como $\mathrm{P}$. Newmark o V. García Yebra. Entre los teóricos de la L: H. E. Wiegand, G. Haensch, R, Werner o F. J. Hausmann. Es significativo que en sus obras principales, cuando existe un índice analítico final, raramente suele aparecer la L (o el D) o la T, respectivamente. 
afín a la T, no central. Wiegand (1987: 15) contempla cuatro campos de aplicación de la que denomina metalexicografía: historia de la L, teoría general de la L, investigación en el uso del diccionario y crítica de DD. En el tercero hay un espacio relevante reservado al uso de los DD (no solo por los traductores, por supuesto), por lo que el espacio que la $\mathrm{L}$ reserva a la $\mathrm{T}$ es, a priori, más destacado en comparación con el que la T reserva a los DD.

La recopilación de una bibliografía sobre las relaciones entre T y L requiere unas pesquisas que, aunque laboriosas, producen resultados más bien escasos, lo que permite concluir sin demasiado esfuerzo que el interés mutuo ha sido hasta el día de hoy muy limitado (como por otra parte reflejaba Varantola en la cita inicial de este trabajo y como también recogen otros autores, p. ej. Wotjak 1997). Hartmann (2004) realizó la primera (y según nuestros datos) única exploración bibliográfica sobre el tema, en pos de estudios de L y de $T$ que hubieran tenido en cuenta cuestiones de tipo interlingüístico. Identifica en primer lugar los que habían aparecido en publicaciones de carácter lexicográfico (revistas, actas de congresos, tesis, etc.), obteniendo un balance bastante exiguo. Pasa a continuación a recorrer el camino inverso, es decir, a localizar contribuciones de utilidad para la $\mathrm{L}$ en publicaciones del campo de la $\mathrm{T}$, con resultados si cabe aún más reducidos.

Otro indicio de ese escaso interés por poner ambas disciplinas en relación lo constituye el número de monografías, de las que se cuentan solamente cinco, desde la pionera y aislada de Snell-Hornby y Pöhl (1989) hasta las más recientes de Sin-wai (2004), Bowker (2006) y San Vicente (2006). A ellas podemos añadir la de Atkins y Varantola (1998) sobre el uso de los DD, si bien no se ocupa solo de $\mathrm{L}$ y $\mathrm{T}$, puesto que a los traductores se añaden los aprendices de lenguas extranjeras. Si examinamos más de cerca estos volúmenes misceláneos nos daremos cuenta de que solo algunas de sus contribuciones tienen un carácter general, metodológico o teórico, mientras el resto se refieren a aspectos concretos aplicados a una lengua o a un par de lenguas. A menudo se trata de obras cuyos capítulos únicamente tratan uno de nuestros dos campos, como sucede en el volumen de Bowker (2006), donde ninguno de sus estudios pone en relación T y L.

Al indagar sobre las causas de este aparente desinterés podríamos preguntarnos, con Humblé (2010: 331):

Serait-ce parce que la relation entre le traducteur et les dictionnaires est tellement évidente que la littérature en traductologie ne semble guère lui accorder de l'importance?

No deja de ser significativo -y será una constante en este trabajo- que la mayor parte de los editores de estos volúmenes y los autores de sus capítulos 
proceden del campo de la L y no del de la T. Una situación análoga se da en el resto de referencias bibliográficas halladas.

\section{Los traductores y los diccionarios}

Uno de los aspectos más estudiados y debatidos es el uso de los DD por parte de los traductores. Hay un elemento común a todos estos estudios: son trabajos que centran su atención en las necesidades de los usuarios de los DD y no en las características de los DD mismos.

El interés por el uso de los DD se manifiesta (Tarp 2009: 276) de manera temprana ya en el lingüista norteamericano Fred W. Householder -quien, en un resumen de un encuentro sobre L celebrado en el lejano 1960, reivindica este enfoque-, y mucho más tarde en autores como Hartmann, Wiegand o Atkins, entre varios otros. Estos lexicógrafos propugnan un cambio de perspectiva en el estudio y en la redacción de DD, de manera que se pase de considerar como centro de interés el producto (el D) a poner el énfasis en sus usuarios.

Así pues, antes de elaborar un D sería indispensable identificar correctamente a los usuarios potenciales de dicha obra y sus necesidades lexicográficas concretas: solo así podremos serles de utilidad. Pero estos buenos deseos chocan a menudo con barreras infranqueables, en particular las editoriales, que por motivos eminentemente prácticos desean obras que sirvan para un número lo más amplio de usuarios finales. Si pensamos en los DD tradicionales (en papel), hemos de ser realistas y comprender que no pueden elaborarse DD (para lo que suelen hacer falta ingentes recursos humanos y económicos) diferenciados para cada grupo de usuarios, si bien es cierto que la irrupción de los medios informáticos en las redacciones lexicográficas pone a nuestro alcance como nunca hasta ahora el cumplimiento del deseo expresado por tantos lexicógrafos (y traductólogos y/o traductores) de crear herramientas lo más flexibles y adaptadas al usuario.

Los usuarios de los DD son muy variados y, además, utilizan dichas obras con finalidades igualmente diversificadas. Hartmann (1989a: 104, 106) ofrece sendos cuadros de los componentes de ambos aspectos (usos y usuarios), que incluyen evidentemente a los traductores (en el grupo de tareas complejas en contexto profesional). No incluye, sin embargo, ninguna distinción ulterior entre traductores profesionales y estudiantes de T, que, como veremos de inmediato, es relevante.

Contamos con un gran número de estudios sobre el uso de los DD, como demuestra la monografía de Welker (2006), que recoge 220 proyectos de investigación publicados entre 1962 y 2006, aunque solo una pequeña parte 
de ellos se centra en lo que hacen los traductores y los estudiantes de T. La gran mayoría de esas investigaciones focalizan su atención precisamente en los estudiantes de T (p. ej., Bowker 1998; Corpas et al. 2001; Hatherall 1984; Mackintosh 1998; Sánchez 2004, 2005; Varantola 1998) y solo unos pocos en los traductores profesionales (por ejemplo, Durán 2010 y Tomaszczyk 1989). Esta preferencia quizá sea debida, como admite Mackintosh (1998: 124), a que se trata de una fuente más sencilla de investigar y a que el posible beneficio en forma de mejoras en los DD repercutirá en quienes más los utilizan, es decir, los propios estudiantes. Omitiremos aquellas investigaciones que, si bien utilizan la T como método para recabar información, lo hacen con sujetos que no guardan una relación directa con el mundo de la T: se trata sobre todo de estudiantes de lenguas extranjeras y, en un caso concreto (Atkins y Varantola 1989), de lexicógrafos participantes en un congreso de L.

No han faltado las críticas hacia los métodos utilizados en estos estudios. Tarp (2009) los clasifica en siete categorías (cuestionarios, entrevistas, observación, protocolos, experimentos, tests y archivos de registro). Observa que todos ellos presentan defectos comunes, como la falta de aleatoriedad en la elección de los sujetos, lo reducido de su número -que lleva a que sus resultados sean estadísticamente poco relevantes-, la formulación de preguntas que predisponen sus resultados (idéntica crítica se encontrará en Hatherall 1984) y otros. Por otra parte, ninguno de estos estudios nos dice nada sobre las situaciones extralexicográficas que dan lugar a las necesidades del usuario, pues se centran casi siempre en la observación mientras se traduce o en el análisis de los resultados. El único método que Tarp (2009: 293) considera fiable, de acuerdo con la teoría funcional en $\mathrm{L}$, es el método deductivo basado en una serie de premisas, que no precisa.

Los estudios basados en el uso que los estudiantes de $\mathrm{T}$ hacen de los DD arrojan unos resultados ciertamente interesantes, aunque muy dispares y difícilmente comparables entre ellos, debido tanto al método de investigación utilizado como a la variedad de aspectos sobre los que se ha indagado. Algunos utilizan el llamado método TAP (think-aloud protocol) (Mackintosh, Varantola), otros utilizan cuestionarios sobre el uso de DD (Corpas et al., Sánchez), otros analizan su comportamiento mientras traducen un texto (Bowker), ${ }^{3}$ añadiendo a veces uno o más cuestionarios (Hatherall), y otros estudian los errores cometidos por los estudiantes (Meyer). Unos se centran en el uso genérico de los DD, otros en el uso del DB general (Meyer) y otros,

3. Es también el procedimiento seguido en Starren \& Thelen (1990), aunque sus planteamientos metodológicos resultan confusos y carece de resultados claros. 
por último, se centran en la traducción especializada (Mackintosh, Bowker, Varantola). En cuanto al número de informantes, varía notablemente, desde el exiguo grupo de 4 estudiantes de Varantola, pasando por los 15 de Mackintosh, los 14 de Bowker, hasta las cifras más consistentes de Corpas et al. (52) y Sánchez (98). En todos los casos se constata un uso predominante del DB: entre el 91,8\% y el 83,7\% para Sánchez, entre el 94,2\% del primer curso y el 73,3\% del último para Corpas et al., o el 59\% de Varantola. En esta última autora encontramos una interesante distinción: en la primera consulta de una búsqueda, la cifra se eleva hasta el $87 \%$, pero cuando los estudiantes (que en este caso están traduciendo a la L2) hacen la segunda se inclinan mayoritariamente por el DM (87\%). Mackintosh es una excepción: sus estudiantes, que traducen un texto especializado a su propia lengua, optaron en su mayoría $(57,9 \%)$ por un diccionario híbrido (bilingualizado).

Estos estudios concuerdan también al constatar que el uso del D (sobre todo el DB) es muy frecuente en el primer año del aprendizaje, pero disminuye (a favor del DM o de otros DD) a medida que se avanza. Las búsquedas en los DB son en su mayoría en pos de equivalentes de $\mathrm{T}$, mientras que en los DM se demandan definiciones, aunque en algunos casos son significativas las búsquedas motivadas por problemas ortográficos (Sánchez 2004). Resulta de interés la distinción que introduce Varantola entre las meras búsquedas de equivalentes (un 55\% en el DB) y las que persiguen simplemente la confirmación de algo ya sabido, que constituye un porcentaje elevado (un $45 \%$ en el DB, un $30 \%$ en el DM).

Durán (2010) lamenta justamente la ausencia de estudios sobre el uso de DD por parte de los traductores profesionales y sus necesidades al respecto, frente a un número mucho más amplio de estudios centrados en los estudiantes de T. Adopta como método la encuesta, con recogida de respuestas a través de internet: elige este método porque ofrece la posibilidad de llegar a un mayor número de sujetos. De hecho, el número de respuestas es ciertamente elevado (402). La mayoría de ellos son traductores (62\%) o intérpretes (14\%) y el resto corresponde a categorías relacionadas. La mayoría (56,4\%) manifestó su preferencia por los recursos en línea. En cuanto al tipo de recursos, al igual que los estudiantes pero con un porcentaje mucho menor, prefieren los bilingües $(39,4 \%)$ frente a los DDMM en L2 o en L1 $(24,1 \%)$; solo un $10,8 \%$ mencionó recursos multilingües, que consideran de calidad inferior, lo que según Durán constituye un toque de atención en toda regla a los terminógrafos. El dato quizá más relevante es el que se deriva de una de las preguntas sobre el recurso preferido ( $i$ Qué tipo de recursos terminológicos usa más para traducir?), que depara alguna sorpresa. Las respuestas fueron, por este orden: DB o 
glosarios especializados (18,9\%), motores de búsqueda tipo Google (16,1\%), bases de datos terminológicas (8,8\%), DM o glosarios especializados (8,6\%), y Wikipedia ( $8,6 \%$ ). Así pues, los DD (como, por otra parte, es bien sabido) no son los únicos recursos a los que acuden los profesionales desde su entorno de trabajo, ya que se han introducido nuevos recursos como Google o Wikipedia, que se utilizan intensivamente a pesar de las constantes críticas en su contra por su falta de fiabilidad. También se preguntó sobre la información que un buen recurso terminológico debería ofrecer. Las respuestas son, en principio, las esperadas, pues entre las informaciones consideradas esenciales se encuentran en los primeros lugares las definiciones claras y concretas y los equivalentes, y les siguen los derivados y compuestos, la especificación del dominio (un aspecto, en cambio, ignorado por los estudiantes), los ejemplos, la información fraseológica, la definición en ambas lenguas en los DDBB y las abreviaciones y acrónimos.

Además del estudio de Durán contamos con el de Tomaszczyk (1989), que se basa en una introspección en su experiencia de uso de los DD como traductor profesional. En el curso de la traducción al inglés (L2) de un manual especializado registró todas sus búsquedas de información. Los resultados más significativos de este experimento son los siguientes: el 81,3\% de las búsquedas (por un total de 691 unidades léxicas) correspondió a términos especializados, el resto al lenguaje común; en el $58 \%$ de las búsquedas de terminología los DD ofrecieron una respuesta satisfactoria, mientras que para las de lenguaje común se elevó al 79,1\%. Del total de búsquedas, el 54\% correspondió a unidades léxicas poco o nada conocidas para el traductor y el $46 \%$ restante fueron meras confirmaciones de lo que el traductor ya sabía: como se indicó antes en Varantola, no hay que perder de vista que el traductor (y también el estudiante avanzado de T) consulta muchas veces los DD no en busca de lo que desconoce sino para confirmar lo que ya sabe. En cuanto al tipo de información buscada: en el $67 \%$ de los casos fueron equivalentes en la L2 en un DB general y en uno técnico general; el 18,4\% correspondieron a terminología general o a un campo específico, en DD especializados; el resto (12\%) fueron consultas en DD de la L2 sobre cuestiones lingüísticas generales. Para Tomaszczyk, a pesar de que los DDMM en L2 son de gran ayuda, considera que los DDBB son la herramienta básica para un trabajo de traducción de este tipo.

Parece, pues, evidente que aún queda mucho por hacer si queremos trazar una imagen mucho más nítida y precisa de las necesidades reales que tienen los traductores (sean estos aprendices, profesionales o de cualquier otra categoría) cuando precisan de la ayuda de los DD. Serían necesarios estudios que 
se valieran también de otros métodos de investigación (además de los más habituales: la encuesta, la observación mientras traducen o el análisis de resultados), que estuvieran basados en una cantidad de materiales relevante (número de informantes, variedad de pruebas, etc.) y que se basaran, además, en avances en los planteamientos teóricos. La inversión de recursos humanos y materiales es necesariamente alta, pero con los medios técnicos actuales debería rebajarse en buena medida. Bien es verdad que en todo ello se ha avanzado mucho, pero el panorama dista aún mucho de presentar un cuadro claro y completo, que sea además de aplicación en el diseño de herramientas lexicográficas adecuadas a las necesidades de traductores y de aprendices de traductor.

\section{Las herramientas lexicográficas al servicio del traductor}

Sobre lo único que parece haber acuerdo entre los estudiosos es que el traductor precisa de herramientas lexicográficas para poder desempeñar eficazmente su trabajo. Sin embargo, más allá de tan genérica afirmación solo encontramos discrepancias. Si bien la mayoría de los autores considera al DB (sea este general o especializado) como la herramienta básica del traductor (y así lo reconocen casi todos los encuestados, como hemos visto en el punto anterior), no han faltado voces que han descalificado estas obras, poniendo en duda su centralidad y considerando de mayor utilidad y efectividad otros tipos de DD. Esta desconfianza hacia el DB, considerado responsable de muchos errores tanto de comprensión como de producción, atañe sobre todo al aprendizaje de lenguas extranjeras y tiene que ver con el rechazo de la traducción como herramienta pedagógica, aunque autores como Corda y Marello (1999) han expresado posiciones más matizadas, destacando que, por un lado, la "prohibición" de hacer uso del DB no impide que se manifiesten interferencias, y, por el otro, en las tareas contrastivas el DB presenta ventajas frente al DM. Estas autoras concluyen sugiriendo acostumbrar al aprendiz a utilizar el tipo de D más adecuado a cada tarea de comprensión, producción o traducción.

Las necesidades que los DD deben satisfacer cuando los traductores los utilizan son muy variadas, en función de varios factores: si se traduce a la Ll o a la L2, si se trata de traducción especializada o no.

En varias de las investigaciones mencionadas en el punto anterior se recogía información sobre qué tipo de herramientas utilizaban tanto los estudiantes de $\mathrm{T}$ como los traductores profesionales. He aquí una somera lista de los tipos de D:

- DD bilingües (generales y especializados).

- DD monolingües (generales y especializados). 
- DD híbridos (bilingualizados).

- DD de sinónimos.

- Enciclopedias.

- Otros diccionarios.

Todas estas obras se pueden presentar en formato papel o en formato electrónico; en este último caso pueden revestir la forma de CD-ROM (y ser utilizadas por tanto en el propio ordenador) o ser consultables directamente a través la red.

Junto a los anteriores encontramos nuevos recursos, todos ellos en línea, que no siempre tienen un carácter netamente lexicográfico, es decir, que no son exactamente diccionarios:

- Bases de datos terminológicas. ${ }^{4}$

- Buscadores (el más popular sin duda es Google).

- Agregadores, es decir, buscadores que realizan consultas simultáneamente en un cierto número de diccionarios.

- Wikipedia.

- Corpus (paralelos o no).

- Gramáticas y estilísticas comparadas. (Wotjak 1997: 123)

Por último, cabe añadir otros recursos también paralexicográficos de los que se valen cotidianamente los traductores en su estación de trabajo, como son las herramientas de las que disponen los programas de edición de texto (correctores ortográficos, diccionarios incorporados, diccionario de sinónimos) o los programas de T. Tarp (2007: 254) se refiere a este tipo de herramientas con un neologismo creado por él: leximatos.

Ante tal abanico de recursos cabe preguntarse si se puede hablar de un diccionario del traductor y si alguna de las obras mencionadas puede ser identificada como tal. En los primeros balbuceos de la L, Zgusta (1971: 216; 1984: 147) asignó al DB la función primaria de diccionario del traductor, al igual que parece hacer Hartmann (1989b: 9). Tras ellos se han multiplicado, de manera más o menos categórica, las dudas sobre su papel como diccionario

4. A propósito de la diferencia entre lexicografía especializada y terminografía, Bergenholtz \& Tarp (2010: 29) opinan: "We still see terminography as a synonym of specialised lexicography. Not all colleagues agree, nor the majority of lexicographers, who see lexicography as a part of linguistics, and most terminographers neither, who claim there are large but unclear differences in relation to specialised lexicography. In reality, it is a discussion about something else, about research funding, about influence and positions at universities, and about defending a position concerning two traditions in making tools to solve exactly the same types of problems." 
del traductor, en dos sentidos: bien por considerar que el DB es poco útil para los traductores (a lo que claramente se oponen autores como Newmark, cuya elocuente cita encabeza este trabajo), bien por colocar junto a él otro tipo de obras igualmente necesarias para el traductor. El concepto de diccionario del traductor aparece en algunos trabajos, pero únicamente Tarp (2007: 231) parece haber ofrecido una definición del mismo:

Un diccionario de traducción es una herramienta cuya función es cubrir las necesidades de información que tengan los traductores en relación con el proceso de traducción.

En su aparente simplicidad esta definición encierra algunas premisas interesantes. Nótese, por ejemplo, que no se hace mención alguna a ningún tipo de diccionario en particular. De hecho, el diccionario del traductor al que aspira este autor es en realidad un diccionario desde el que se puede acceder a múltiples fuentes de información (Tarp 2007: 256).

Hay consenso al considerar que un solo tipo de D no es suficiente para el trabajo del traductor. Iamartino (2006: 106), por ejemplo, piensa que no hay un único D del traductor, ya que hay muchos tipos de traductores y de traducciones, y por tanto muchos tipos posibles de D del traductor. Pero la preferencia dada a unos u otros marca diferencias entre los especialistas. Por otra parte, está extendida también la idea de que cualquier obra lexicográfica, por muy deficiente que sea, puede resultar en un momento dado de utilidad para el traductor e incluso justificar su adquisición (Newmark 1998; Piotrowski 1994: 118). Por ejemplo, algunos DD unánimemente considerados como insatisfactorios y superados, pueden contener informaciones enciclopédicas útiles desde un punto de vista diacrónico.

Como hemos visto antes, el DB es el preferido por casi todos los estudiantes de T y también por los traductores, pero no faltan las voces discordantes. Mackintosh (1998) considera que el instrumento ideal (en su caso para la traducción especializada a la L2) es el D híbrido o bilingualizado. Fraser (1999) admite su uso como simple punto de partida para el buen traductor, para obtener pistas y sugerencias, mientras que lo considera un instrumento poco útil e incluso peligroso en manos de los malos traductores. Piotrowski (1995) desaconseja también su uso por parte de los traductores (no por parte de otros, como los turistas o los estudiantes de L2); en su opinión, el DB, para ser un auténtico diccionario de traducción, debería contener un número de equivalentes prácticamente ilimitado, que cubriera una cantidad infinita de contextos, lo que es imposible; aconseja el uso del DM y de un diccionario de sinónimos para traducir a la Ll. Roberts (1990) se hace eco de las críticas que los traductores hacen de los DD y habla de un sentimiento de "frustración" 
(idéntico sentimiento se recoge en Varantola 1998: 180). Roberts no critica directamente al DB (pues considera misión casi imposible que satisfaga las necesidades de todos los tipos de traductores), sino su uso por parte de muchos traductores y de la práctica totalidad de los estudiantes de T, que se lanzan ciegamente sobre él en busca de soluciones en cualquiera de las fases en las que se desarrolla el proceso traductor.

Otro recurso reivindicado por ciertos autores es el de los corpus. Bowker (1998), basándose en un estudio empírico sobre un grupo de estudiantes (algunos de ellos utilizaron corpus, mientras que otros dispusieron de otros DD al realizar una traducción especializada a la L1), observó que quienes habían utilizado un corpus de textos especializados obtuvieron mejores resultados, por lo que considera aconsejable su uso, a pesar de que (como ella misma reconoce) hay pocos disponibles. ${ }^{5} \mathrm{~A}$ esto podríamos añadir que la disponibilidad de recursos (corpus, pero también DB especializados, bases de datos terminológicas, diccionarios híbridos o incluso buenos DDBB) es más o menos limitada en función de la lengua o lenguas implicadas. Como no podía ser de otra manera, Hartmann (2004), padre de la textología contrastiva y artífice de una conocida clasificación de los corpus paralelos, propugna su uso en la elaboración de DD (también de manera decidida Wotjak 1997: 115). También Tomaszczyk (1989) contempla el uso de corpus bilingües de calidad (del tercer tipo en la clasificación de Hartmann, es decir, los corpus compuestos por textos que no son traducciones unos de otros, sino que son independientes aunque comparables por tratar del mismo tema) para que el traductor aprenda sobre campos de especialidad que le son poco familiares.

Las numerosas opiniones vertidas sobre el uso de las herramientas lexicográficas en T podrían dividirse en dos grupos: las de quienes observan el proceso de $\mathrm{T}$ como un todo (o bien se centran en herramientas concretas) y la de quienes desglosan dicho proceso en sus diferentes fases, para cada una de las cuales se aconseja el uso de herramientas diferentes.

Entre las primeras está la de Iamartino (2006), que analiza la validez de cinco DDBB italiano-inglés a la hora de traducir varias categorías léxicas, entre las que se encuentran, además de algunas previsibles (tecnicismos, colocaciones y unidades fraseológicas, palabras culturales, etc.), otras quizá menos

5. A conclusiones similares llega Zucchini (2011) en un estudio experimental sobre el uso de distintos recursos en tareas de traducción especializada: los DD se revelaron útiles para colmar lagunas lingüísticas, el glosario terminológico específico resultó ser el recurso más completo, mientras que el corpus comparable utilizado en la elaboración del glosario pudo satisfacer buena parte de las necesidades de información sobre usos y fraseología, permitiendo mejores resultados. 
previsibles, como el propio léxico de base (no marcado) de la lengua, que a menudo se piensa erróneamente que no plantea problemas. Entre sus conclusiones destaca que los equivalentes propuestos en el DB serán más o menos adecuados si se traduce a la L1 o a la L2 y que los DD actuales deben mejorar mucho añadiendo abundantes remisiones internas y también etiquetas y notas de uso, si realmente quieren ser de utilidad para el traductor.

Otros autores detectan necesidades diferentes en las diferentes fases de la traducción y, por tanto, poder disponer en cada una de ellas de herramientas diferenciadas. Roberts (1990) propone lo siguiente:

- Análisis del texto: el traductor suele acudir al DB en busca de aclaraciones sobre el significado de las unidades léxicas, pero a menudo queda decepcionado por la falta de indicaciones semánticas junto a los equivalentes, además de la habitual pobreza al recoger unidades fraseológicas y palabras del léxico marcado (regional, etc.), por lo que será de mayor ayuda el DM.

- Transferencia del texto a la L2: lamenta que a menudo no se encuentre el equivalente adecuado, que a veces figura solo en los ejemplos. Falta, pues, una separación clara de los sentidos, indicaciones y notas de uso, mención de los actantes, etc.

- Revisión de la traducción: suele utilizarse el DB general, pero normalmente es de poca utilidad, por los mismos motivos aducidos para la fase anterior.

Tarp (2007), que se refiere en todo momento a la traducción especializada, distingue tres fases, a las que hay que añadir otras dos (prefase y postfase):

- Prefase: será de gran utilidad, si el traductor no domina el tema, una breve introducción al campo de especialidad, en ambas lenguas.

- Recepción: las necesidades podrán ser cubiertas con un DM de la lengua de partida o bien con un DB.

- Transferencia: se usará necesariamente un DB que contenga abundante información sobre equivalentes y también sobre colocaciones. El DB tendrá que tratar tres tipos de léxico: a) léxico general (se incluirán diferenciadores semánticos junto a los equivalentes); b) términos de una especialidad que no varía de un país a otro (p. ej., la biología); c) términos que sí cambian (p. ej., el derecho: en este caso el traductor necesitará más información adicional).

- Producción: si se trata de problemas que ya se han planteado en la fase anterior se recomienda el DB, mientras que si se trata de cuestiones nuevas será más adecuado el DM de la lengua de llegada. 
- Postfase o fase de revisión: recoge todo lo de las anteriores; dado que se centra en la lengua de llegada las herramientas más útiles serán un DM de dicha lengua o un DB que parta también de ella.

Se plantea a continuación una pregunta muy pertinente: ¿qué solución podemos encontrar frente a este complejo conjunto de necesidades? Habría dos, una dispersa (la consulta de diferentes fuentes, diccionarios, etc.; es la que parece predominar hasta hoy) y otra integrada (un único D en el que se localizarían diferentes soluciones lexicográficas): se decanta evidentemente por la segunda.

Se hacen necesarias otras distinciones en cuanto al uso de los DD. Así, no es lo mismo traducir a la propia lengua (L1) que a la lengua extranjera (L2). En el segundo caso el traductor necesita un caudal de información de todo tipo muy superior al primero; y si nos referimos a un D para el estudiante de T las necesidades aumentan más todavía (Wotjak 1997: 114); entonces, además de los DD podrá ser de utilidad el recurso a corpus bien confeccionados (como reclamaban también Bowker, Hartmann y Tomaszczyk). El traductor especializado tiene unas necesidades diferentes de las de quien traduce textos sin un alto grado de especialización.

En el panorama actual de la $\mathrm{L}$ es ineludible distinguir entre dos grupos de DD, en función de su soporte material: DD en papel y DD electrónicos. Aunque el salto cualitativo que se ha producido en todos los órdenes al aparecer los segundos ha sido sin duda enorme, la L electrónica, a pesar de sus ya más de 20-30 años de existencia, no ha sacado aún todo el partido posible a su potencialidad, fundamentalmente porque se ha mantenido (al menos en los productos comerciales más difundidos) muy ligada a las versiones en papel, que son su punto de partida. De hecho, una de las críticas más generalizadas a estos DD electrónicos (sean en CD-ROM o de consulta en línea) es que a menudo son meras transposiciones de sus versiones en papel, con muy poca información añadida (más allá, por ejemplo, de la pronunciación en voz alta de sus lemas). Es cierto que poseen una capacidad de búsquedas y de navegación infinitamente mayor respecto a la versión en papel, pero no se aprovechan características tales como la inexistencia de límites de espacio (para incluir más informaciones, más ejemplos, más notas de uso, gráficos, fotografías, remisiones a corpus, etc.) y la no obligación de seguir la rígida organización de la macroestructura y la microestructura de sus antecedentes en papel. Los DD concebidos expresamente para su consulta en la red son los que parecen estar superando estos límites y acercándose a un desiderátum ampliamente sentido de individualización o personalización de los DD, es 
decir, que el D pueda ofrecer respuestas diferenciadas en función del perfil de usuario y del tipo de petición de información que realice. De este presupuesto parten, por ejemplo, los DD elaborados en los últimos años por el Centro de Lexicografía de la Universidad de Aarhus y sus colaboradores.

Fuertes y Nielsen (2012) estudian específicamente tres DD terminológicos en línea (Cercaterm, DiCoInfo, EuroTermBank) con la finalidad de comprobar su adecuación a los postulados de la teoría funcional lexicográfica y por tanto su utilidad en la traducción especializada. El principal problema que detectan es su falta de flexibilidad y de adecuación al usuario, puesto que no contemplan una posible individualización en su uso.

La situación en el campo de la L electrónica dista, pues, de ser idílica y son muchos aún los obstáculos de todo tipo que se deben superar. Dejando a un lado cómo deberían ser estos DD en el futuro, los actuales son blanco de numerosas críticas. Pastor y Alcina (2010) analizan un gran número de ellos y ofrecen una clasificación detallada de sus técnicas de búsqueda. Afirman (y en ello concuerdan otros autores) que los usuarios de estos DD no les sacan todo el partido posible por dos motivos: porque no hacen más sencillo el acceso a sus datos y porque los usuarios no saben muy bien cómo acceder a ellos. Podemos añadir las objeciones de Tarp (2007: 254): a) para el usuario es a menudo difícil determinar cuál es su necesidad concreta en un momento dado y por tanto hacer una búsqueda rápida y eficaz; b) muchos datos de los artículos prefabricados (como los del $\mathrm{D}$ en papel) resultan totalmente irrelevantes; y c) los artículos prefabricados no pueden contener la información necesaria para satisfacer búsquedas complejas. También Forget (1999) se ocupa del tema, aunque en este ámbito el paso del tiempo resulta inmisericorde y los casi 15 años transcurridos, además de los avances actuales en la informática y (algo menores) en la L informatizada, han convertido este amplio estudio en poco relevante en la actualidad.

Ciertos problemas en los DD actuales están directamente ligados con cuestiones de tipo práctico, más concretamente con las decisiones de las editoriales. La publicación de DD suele suponer la movilización de cuantiosos recursos humanos y económicos, de ahí que estos productos se conciban con la lógica finalidad de conseguir un beneficio económico. Esto choca con los deseos, justificados, de muchos lexicógrafos (y también de muchos traductores) de encontrar en el mercado DD que se ajusten más a sus postulados (en el caso de los lexicógrafos, tanto teóricos como prácticos) y sus necesidades (en el caso de los traductores). Así, se ha pedido a menudo (Marello 1989: 18; Wotjak 1997: 115) que los DDBB aparecieran en cuatro versiones diferentes, en función tanto de la combinación lingüística (L1 y L2) como de su 
función (codificación y descodificación). El traductor especializado desearía mayor información junto a los equivalentes en la L2 cuando tiene que realizar traducciones inversas, así como información contrastiva que señale en qué se distinguen los conceptos a los que remiten los términos de las lenguas implicadas (Werner 2002). Pero las editoriales no suelen atender estas demandas y crean productos que, si bien son a menudo de buena calidad, no pueden satisfacer de manera igual a todos los usuarios. El uso generalizado de la informática en la elaboración de los DD actuales debería facilitar en un futuro no muy lejano la existencia de obras que se adapten lo más posible a sus usuarios, y no al revés, como a menudo sucede ahora.

Mientras que las críticas al papel que juegan los DD en la T son más o menos generalizadas, a la hora de proponer mejoras o alternativas las voces son mucho menos numerosas. Las propuestas de soluciones van encaminadas en tres direcciones: a) las que proponen nuevos tipos de herramientas lexicográficas (como el Centro de Aarhus que ya hemos señalado); b) las que proponen mejoras en las actuales; y c) las que proponen mejorar la consulta de los DD mediante su adecuada enseñanza a los estudiantes de $\mathrm{T}$.

Nos centraremos ahora en el último punto, que debería ser el más sencillo de aplicar, aunque en realidad no siempre es así. Roberts (1992), una de las autoras más críticas con los DD, en particular los bilingües, considera que el problema radica en el mal uso que los estudiantes hacen de los DD, puesto que, frente a unos productos cada vez mejores y más completos, encontramos unos usuarios (los estudiantes) que no saben buscar en ellos lo que necesitan y que se dirigen normalmente a ellos de manera ciega e impulsiva. Se hace necesario enseñar el uso correcto del $\mathrm{D}$, lo que, en su opinión, debería, pues, encontrar cabida en alguna de las asignaturas de documentación que suelen figurar en los planes de estudios de T y es muy importante que sea impartida por un profesor familiarizado con la $\mathrm{T}$, puesto que a menudo lo hace un bibliotecario que carece de tales conocimientos, restándole así buena parte de su utilidad. Para Roberts (1992: 53 ss.) esta enseñanza se ha de basar en la consecución de cuatro objetivos: a) familiarización con los diferentes tipos de unidades léxicas; b) familiarización con los diferentes tipos de DD; c) familiarización con los formatos de entrada; y d) ilustración de las vías para combinar análisis del texto, traducción y consulta del D. Estas cuatro categorías representan cuatro problemas principales en lo que respecta a la traducción por parte de los estudiantes: a) saber qué buscar en un D; b) saber dónde buscar la información léxica; c) saber cómo interpretar la información léxica ofrecida; d) saber cuándo y cómo consultar DD durante el proceso de 
traducción. Para esta autora el aspecto más interesante es el último, ya que engloba a los anteriores.

No han faltado en los últimos decenios propuestas de elaboración de nuevos tipos de DD diseñados expresamente para los traductores, aunque en pocos casos se han plasmado en productos concretos. Marello (1989: 120-122), por ejemplo, invocando la colaboración entre lexicógrafos y traductores (sean estos estudiantes o profesionales) sugiere la elaboración de un nuevo D activo fruto de la experiencia y sensibilidad de ambos.

Rogers y Ahmad (1998) realizaron un certero análisis y pronosticaron para un futuro no demasiado lejano la realización de un diccionario del traductor realmente adecuado:

We would like to argue that the translator's dictionary of the future will be a more dynamic concept than that of the specialised paper-based dictionary of today or its replication on computer systems, often misleadingly represented as machine-readable dictionaries, terminology databases or lexical resource databases. The new dictionary will allow translators not only to draw on electronically-stored data-bases of terms with smarter semantically-relevant navigational paths, it will also support them in creating their own data-bases or simply help them to solve their problems in an ad hoc way by reference to large electronic corpora of text, a source of context-sensitive reference for language use. An integrated interface allowing access to a range of sources, including the Internet, following in the increasingly familiar workbench approach [...] would allow the user/translator to extend and complement standard sources.

Como indicamos en otro lugar de este artículo, una de las pocas propuestas que se ha plasmado en productos tangibles es la de los especialistas del Centro de Lexicografía de Aarhus, que ha producido ya un abanico de DD especializados de nuevo cuño que parten de varios presupuestos, en particular que el traductor especializado no solo necesita términos y sus traducciones, sino también información sobre aspectos textuales y pragmáticos y también conocimientos sobre la materia de que se trate, todo ello en dos lenguas, entre otras características (cfr. p. ej., Nielsen 2010).

Un campo ciertamente particular, que excede los confines que nos hemos marcado, atañe a la elaboración de lexicones, diccionarios informatizados o diccionarios máquina para la traducción automática. Se trata de un sector bastante desarrollado que, sin embargo, a fecha de hoy ofrece aún resultados limitados e incluso modestos, sobre todo si los comparamos con las expectativas que se despertaron en los inicios de este campo de investigación. Citamos, a mero título de ejemplo, a Guest y Mairal (2007), Jiménez (2001) y Lépinette (1990, 1994), entre muchos otros. 


\section{El lexicógrafo como traductor, el traductor como lexicógrafo}

Hasta ahora hemos estudiado la relación entre T y L casi en exclusiva en una dirección: el uso de los DD por parte de los traductores. Pero, ¿es posible recorrer la dirección opuesta? Es decir, ¿puede la T o el traductor resultar de utilidad para la L o para el lexicógrafo? Y surge otra pregunta: ¿pueden reunirse en un mismo individuo las figuras del traductor y del lexicógrafo? O dicho de otro modo: ¿es el lexicógrafo (bilingüe) en realidad un traductor?

Hartmann (2004: 11) es uno de los pocos autores que da alguna pista sobre lo que la $\mathrm{T}$ puede hacer por la $\mathrm{L}$, aunque luego no desarrolle la idea:

Translation is relevant to lexicography in two ways: as supplier of translation equivalents to be included in the bilingual dictionary and as consumer of information made available by lexicographers to professional translators.

Así pues, Hartmann (1994; 1989b: 17) es partidario del uso de textos paralelos de un cierto tipo (los dos primeros tipos de su conocida clasificación, es decir, los que son traducciones unos de otros o de ambos respecto a un texto común en una tercera lengua) como fuente de equivalentes de traducción y de colocaciones para que el lexicógrafo las utilice en la redacción de DDBB, pues está firmemente convencido de las virtudes de tal proceder, que puede llevar a superar algunas de las limitaciones que presentan los equivalentes en los DDBB, que suelen obtenerse teniendo en cuenta los contextos más comunes y por tanto obviando otros muchos, quizá menos comunes pero necesarios para el traductor.

Humblé (2010), por su parte, y en relación con otra de las preguntas realizadas antes, tras constatar -como hemos hecho ya en este trabajo- la indiferencia en el campo de la $\mathrm{T}$ hacia la $\mathrm{L}$, reflexiona sobre las profundas relaciones que se establecen entre el lexicógrafo bilingüe y la T. Afirma, por ejemplo -y se cura en salud advirtiendo de una posible reacción adversa de los traductólogos- que el lexicógrafo bilingüe es en realidad un traductor, no solo porque la actividad de traducción es habitual en él, sino porque la elaboración de un DB supone una gigantesca empresa de traducción, puesto que se trata de traducir en otra lengua todo el caudal léxico de una lengua o al menos buena parte de él. Reconoce, por supuesto, que las traducciones que ofrece el lexicógrafo no son del mismo tipo de las del traductor, como ya sabemos. Subraya, sin embargo, que hay otra parte del artículo lexicográfico en la que el lexicógrafo se iguala con el traductor: los ejemplos. En este caso lexicógrafo y traductor se encuentran en situaciones análogas frente a problemas similares. 


\section{Conclusión}

El llamamiento para la publicación de este número sugería una serie de posibles temas, algunos más explorados que otros. Parte de ellos han sido recogidos por los autores que han enviado sus propuestas, como por ejemplo: herramientas electrónicas y traducción (artículo de Durán), DD en los diferentes tipos de traducción (jurídica, técnica, etc.) (Fuertes, Nielsen y Bergenholtz; Corpas y Roldán; Gallego), DD específicos para traductores (Buendía y Faber; o el artículo de Tarp sobre el diccionario del traductor). No han faltado artículos que caen fuera de los temas previstos, pero que revisten gran interés (Sánchez Martín; Vaxelaire). Otros temas han quedado inexplorados, por ejemplo: otros DD (enciclopédicos, de sinónimos, etc.) en traducción, el diccionario en la didáctica de la traducción, uso de corpus paralelos (de traducciones o comparables) en la elaboración de DD bilingües. La elección de los autores coincide en líneas generales con los temas que resultan más habituales en el panorama bibliográfico que hemos intentado trazar, aunque no del todo: por ejemplo, la enseñanza del uso del diccionario a los aprendices de traductor es un tema que cuenta con una cierta bibliografía, pero que aquí está ausente.

El interés por la disciplina hermana se concreta en modos diferentes, tanto cualitativa como cuantitativamente. Los lexicógrafos han sentido preocupación por cubrir de la mejor manera posible las necesidades de los traductores. Los traductores han lamentado a menudo la inadecuación de los instrumentos lexicográficos respecto a sus necesidades, pero sin realizar a menudo sugerencias de mejora concretas a los lexicógrafos. Ha faltado, pues, un diálogo más fluido entre ambas partes, sin duda condenadas a entenderse. En este sentido cabe recordar que el interés ha sido siempre mucho mayor por parte de los lexicógrafos. No se da una bidireccionalidad en los estudios que implican a las dos disciplinas. En efecto, los estudios sobre el uso de diccionarios en Traducción constituyen una mayoría aplastante. Los estudios sobre el uso de la Traducción o las traducciones en Lexicografía son muy escasos.

Esperamos que los artículos aquí recogidos puedan aportar un grano de arena a esta gran tarea colectiva, y contribuir a abrir nuevos caminos

\section{Bibliografía}

AtKINS, B. T. Sue (ed.) (1998) Using dictionaries. Studies of dictionary use by language learners and translators. Tübingen: Max Miemeyer.

BOWKER, Lynne. (1998) "Using Specialized Monolingual Native-Language Corpora as a Translation Resource: A Pilot Study." Meta 43:4, pp. 631-651.

BOWKER, Lynne (ed.). (2006) Lexicography, terminology, and Translation: textbased studies in honour of Ingrid Meyer. Ottawa: Université d'Ottawa. 
CORDA, Alessandra \& Carla Marello. (1999) Insegnare e imparare il lessico. Torino: Paravia.

CORPAS PASTOR, Gloria; Jorge Leiva Rojo \& M $\mathrm{M}^{\mathrm{a}}$ José Varela Salinas. (2001) "El papel del diccionario en la formación de traductores e intérpretes: análisis de necesidades y encuestas de uso." En: Ayal, M. (ed.) 2001. Diccionarios y enseñanza. Alcalá de Henares: Servicio de Publicaciones de la Universidad de Alcalá, pp. 239-273.

DURÁN MUÑOZ, Isabel. (2010) "Specialized lexicographical resources: a survey of translators' needs." En: Granger, Sylviane \& Magali Paquot (eds.) 2010. eLexicography in the $21^{\text {st }}$ century: New Challenges, new applications. Proceedings of ELEX2009. Cahiers du Cental, vol. 7. Lovaine-La-Neuve: Presses Universitaires de Louvain, pp. 55-66.

FORGET, Nadine. (1999) Les dictionnaires électroniques dans l'optique de la traduction. Ottawa: Université d'Ottawa. Thèse de maîtrise. École de traduction et d'interprétation. Versión electrónica: http://aixl.uottawa.ca/ etithese/nforg/ frmain.htm

FRASER, Janet. (1999) "The translator and the word: the pros and cons of dictionaries in translation." En: Anderman, G. \& M. Rogers. (eds.) 1999. Word, text, translation. Liber amicorum for Peter Newmark. Clevedon \& Philadelphia: Multilingual Matters, pp. 25-34.

Fuertes Olivera, Pedro A. \& Sandro Nielsen. (2012) "Online dictionaries for assisting translators of LSP texts: The Accounting Dictionaries." International Journal of Lexicography 25:2, pp. 191-215.

GUEST, Elizabeth \& Ricardo Mairal Usón. (2007) "Building a computational lexicon for automatic translation: A preliminary discussion." En: Fuertes Olivera, Pedro A. et al. (eds.) 2007. Problemas lingüísticos en la traducción especializada. Valladolid: Universidad de Valladolid, pp. 197-226.

HARTMANN, Reinhard Rudolf Karl. (1989a) "Sociology of the Dictionary User: Hypotheses and Empirical Studies." En: Hausmann, Franz J.; O. Reichmann; H. E. Wiegand \& L. Zgusta (eds.) 1989. Worterbucher. Dictionaries. Dictionnaires. Ein internationales Handbuch zur Lexikographie. Berlin-Nueva York: de Gruyter, pp. 102-111.

HARTMANN, Reinhard Rudolf Karl. (1989b) "Lexicography, translation and the so- called language barrier.” En: Snell-Hornby, Mary \& Esther Pöhl (eds.) 1989. Translation and Lexicography. Papers read at the Euralex Colloquium held at Innsbruck 2-5 July 1987. Amsterdam: John Benjamins B.V. et al., pp. 9-20.

HARTMAnN, Reinhard Rudolf Karl. (1994) "The Use of Parallel Text Corpora in the Generation of Translation Equivalents for Bilingual Lexicography". En: Martin, W. et al. (eds.) 1994. EURALEX 94 Proceedings. Amsterdam: John Benjamins, pp. 291-297. 
HARTMAnN, Reinhard Rudolf Karl. (1999) "Thematic Report 2. Case Study: The Exeter University Survey of Dictionary Use." En: Hartmann, R. R. K. (ed.) 1999. Dictionaries in Language Learning. Recommendations, National Reports and Thematic Reports from the TNP Sub-Project 9: Dictionaries. Berlin: Freie Universität Berlin, pp. 36-52.

HaRtMann, Reinhard Rudolf Karl. (2004) "Lexicography and translation." En: Sin-wai, Chan (ed.) 2004. Translation and Bilingual Dictionaries. Tübingen: Max Miemeyer, pp. 7-21.

HATHERALL, Glyn. (1984) "Studying dictionary use: Some findings and proposals.” En: Hartmann, R.R.K. (ed.) 1984. LEXeter '83 Proceedings. Papers from the International Conference on Lexicography at Exeter, 9-12 September 1983. Tübingen: Max Niemeyer, pp. 183-189.

Humblé, Philippe. (2010) "Dictionnaires et traductologie : le paradoxe d'une lointaine proximité." Meta 55:2, pp. 329-337.

IAMARTINO, Giovanni. (2006) "Dal lessicografo al traduttore: un sogno che si realizza?" En: San Vicente, Félix (ed.) 2006. Lessicografia bilingue e traduzione: metodi, strumenti, approcci attuali. Monza: Polimetrica, pp. 101-132.

JimÉNEZ Ríos, Enrique. (2001) "O papel da lexicografía nas novas tecnoloxías aplicadas á tradución." Viceversa 6, pp. 37-58.

LÉPINETTE, Brigitte. (1990) "Lexicographie bilingue et traduction." Meta 35:3, pp. 571-581.

LÉPINETTE, Brigitte. (1994) "La lexicografía bilingüe no convencional. Propuesta para la construcción de una base de datos contrastivos destinada a la traducción automática." En: Charlo Brea, Luis (ed.) 1994. Reflexiones sobre la Traducción: Actas del Primer Encuentro Interdisciplinar "Teoría y Práctica de la Traducción”. Cádiz: Universidad de Cádiz, pp. 345-354.

MACKInTOSH, Kristen. (1998) "An Empirical Study of Dictionary Use in L2-L1 Translation.” En: Atkins, B. T. Sue (ed.) 1998 Using Dictionaries: Studies of Dictionary Use by Language Learners and Translators. Tübingen: Niemeyer, pp. 121-149.

MARELlO, Carla. (1989) Dizionari bilingui, con schede sui dizionari italiani per francese, inglese, spagnolo, tedesco. Bologna: Zanichelli.

MEYER, Ingrid. (1988) "The General Bilingual Dictionary as a Working Tool in Thème." Meta 33:3, pp. 368-376.

Newmark, Peter. (1998) A Textbook of Translation. London: Prentice Hall.

NiELSEN, Sandro. (2010) "Specialized Translation Dictionaries for Learners." En: Fuertes-Olivera, Pedro A. (ed.) 2010. Specialized Dictionaries for Learners. Berlin \& New York: De Gruyter, pp. 69-82.

PASTOR, Verónica \& Amparo Alcina. (2010) "Search Techniques in Electronic Dictionaries: A Classification for Translators." International Journal of Lexicography 23:3, pp. 307-354. 
PiOTROWski, Tadeusz. (1994) Problems in bilingual lexicography. Wroclaw: Wydawnictwo Uniwersytetu Wroclawskiego.

RoberTS, Roda P. (1990) “Translation and the Bilingual Dictionary." Meta 35:1, pp. 74-81.

ROBERTS, Roda P. (1992) "Traslation pedagogy: strategies for improving dictionary use.” Traduction, Terminologie et Rédaction 5:1, pp. 49-76.

RogERS, Margaret \& Khurshid Ahmad. (1998) "The Translator and the Dictionary: Beyond Words.” En: Atkins, B. T. Sue (ed.). 1998. Using Dictionaries: Studies of Dictionary Use by Language Learners and Translators. Tübingen: Niemeyer, pp. 193-204.

SAlmon, Laura. (2003) Teoria della traduzione. Storia, scienza, professione. Milano: A. Vallardi.

SAN VicEnTE, Félix (ed.) (2006) Lessicografia bilingue e traduzione: metodi, strumenti, approcci attuali. Monza: Polimetrica.

SÁnCHEZ RAMOS, M. del Mar. (2004) "Estudio experimental sobre el uso del diccionario como herramienta para el traductor: hacia una descripción de necesidades." En: Ortega, E. (ed.) 2004. Panorama actual de la investigación en Traducción e Interpretación. Granada: Atrio, pp. 477-486.

SÁnCHEZ RAmos, M. del Mar. (2005) "Research on Dictionary Use by Trainee Translators." Translator Journal 9:2. Versión electrónica: http://translationjournal.net/journal/32dictuse.htm

SiN-WAI, Chan (ed.) (2004) Translation and Bilingual Dictionaries. Tübingen: Max Miemeyer.

SNell-Hornby, Mary \& Esther Pöhl (eds.) (1989) Translation and Lexicography. Papers read at the Euralex Colloquium held at Innsbruck 2-5 July 1987. Amsterdam: John Benjamins B.V. et al.

StARren, Peter \& Marcel Thelen. (1990) "General dictionaries and students of translation: A report on the use of dictionaries in the translation process." En: Magay, T. \& J. Zigàny (eds.) 1990. BudaLEX '88 proceedings. Budapest: Akadémiai Kiadó.

TARP, Sven. (2004) "How can dictionaries assist translators?" En: Sin-wai, Chan (ed.) 2004. Translation and Bilingual Dictionaries. Tübingen: Max Miemeyer, pp. 23-38.

TARP, Sven. (2007) “¿Qué requisitos debe cumplir un diccionario de traducción del siglo 21?" En: Fuertes Olivera, Pedro A. (ed.) 2007. Problemas linguísticos en la traducción especializada. Valladolid: Universidad de Valladolid, pp. 227-256.

TARP, Sven. (2008) Lexicography in the borderland between knowledge and nonknowledge. Tübingen: Max Niemeyer.

TARP, Sven. (2009) "Reflections on Lexicographic User Research." Lexikos 19, pp. 275-296. 
TOMASZCZYK, Jerzy. (1989) "L1-L2 Technical Translation and Dictionaries." En: Snell-Hornby, Mary \& Esther Pöhl (eds.) 1989. Translation and Lexicography. Papers read at the Euralex Colloquium held at Innsbruck 2-5 July 1987. Amsterdam: John Benjamins B.V. et al., pp. 177-186.

VAN Hoof, Henri. (1991) Histoire de la traduction en Occident. Paris-Louvaine-laNeuve: Duculot.

Varantola, Krista. (2002) "Use and Usability of Dictionaries: Common Sense and Context Sensibility?" En: Corréard, Marie-Hélène (ed.) 2002. Lexicography and Natural Language Processing. A Festschrift in Honour of B.T.S. Atkins. Euralex, pp. 30-44.

WeLKER, Herbert Andreas. (2006) O uso de dicionários: Panorama geral das pesquisas empiricas. Brasilia: Thesaurus.

WERNER, Reinhold. (2002) "El problema de la equivalencia en los diccionarios bilingües especializados". En: Ahumada, Ignacio (ed.) 2002. Diccionarios y lenguas de especialidad. V Seminario de L Hispánica. Jaén, 21 al 23 de noviembre de 2001. Jaén: Universidad de Jaén, pp. 3-20.

WiEGAND, Herbert E. (1984) "On the Structure and Contents of a General Theory of Lexicography." En: Hartmann, R. R. K. (ed.) 1984. LEXeter '83 Proceedings. Papers from the International Conference on Lexicography at Exeter, 9-12 September 1983. Tübingen: Max Niemeyer, pp. 13-30.

WotJAK, Gerd. (1997) "Reflexiones acerca de un diccionario para traductores." En: Vega, Miguel Ángel \& R. Martín-Gaitero (eds.) 1997. La palabra vertida. Madrid: Universidad Complutense, pp. 111-124.

ZGusta, Ladislav. (1971) Manual of Lexicography. La Haya \& París: Mouton.

ZGUSTA, Ladislav. (1984) "Translational equivalence in the bilingual dictionary." En: Hartmann, R. R. K. (ed.) 1984. LEXeter '83 Proceedings. Papers from the International Conference on Lexicography at Exeter, 9--12 September 1983. Tübingen: Max Niemeyer, pp. 147-154.

ZUCCHINI, Luisa. (2011) "Riflessioni terminologiche e lessicologiche sul glossario 'Linguaturismo' e la sua applicazione nella didattica della traduzione". En: Liverani, E. \& Canals, J. (eds.) 2011. El discurso del turismo. Aspectos lingüísticos $y$ variedades textuales. Trento: Tangram, pp. 17-34.

\section{NOTAS BIOGRÁFICAS / BIONOTES}

\section{Cesáreo Calvo Rigual}

Cesáreo Calvo Rigual es Catedrático de Filología Italiana en la Universitat de València. Doctor en Filología (1993) con una tesis doctoral sobre "El lèxic de la traducció italiana del Tirant lo Blanch (1538)". Ha publicado numerosos 
estudios en los siguientes campos: lexicografía monolingüe (italiana) y bilingüe (italiano-español e italiano-valenciano), lingüística contrastiva italianoespañol e historia de la traducción de obras italianas al español y al catalán. En este último campo ha contribuido a la creación y mantenimiento del Proyecto Boscán (Catálogo de las Traducciones Españolas de Obras Italianas, hasta 1939). Ha traducido al español obras de Pietro Aretino y de Giovanni della Casa además de La veneciana de anónimo veneciano del siglo XVI. Es autor, junto a Anna Giordano, del Diccionario italiano Herder (1995, 2005 y 2010).

Cesáreo Calvo Rigual is Professor of Italian at the University of Valencia. He received his PhD in Languages and Literatures (1993) with a dissertation entitled El lèxic de la traducció italiana del Tirant lo Blanch (1538). He has published numerous studies in the following areas: monolingual lexicography (Italian) and bilingual lexicography (Italian-Spanish and Italian-Catalan), Italian-Spanish contrastive linguistics, and history of the translation of Italian works into Spanish and Catalan. In this latter field, he has contributed to the creation and development of the project Proyecto Boscán (Catalogue of Spanish Translations of Italian Works, until 1939). He has translated works by Pietro Aretino and Giovanni della Casa into Spanish, as well as La venexiana, by an anonymous Venetian author of the 16th century. He coauthored, with Anna Giordano, the Herder Italian Dictionary (1995, 2005 and 2010).

\section{Maria Vittoria Calvi}

Maria Vittoria Calvi, catedrática de Lengua española de la Universidad de Milán, tiene una larga experiencia en la investigación y en la docencia universitaria; es vicepresidenta de la Asociación de Hispanistas Italianos (trienio 2010-14) y es miembro del CERLIS (Centro di Ricerca sui Linguaggi Specialistici) de la Universidad de Bérgamo. Es autora de numerosos estudios de lingüística española, con especial atención en la lingüística contrastiva de español e italiano y la enseñanza-aprendizaje de lenguas afines. Entre sus líneas de investigación más recientes, también cabe destacar el contacto entre español e italiano en contextos migratorios. En campo literario, sus trabajos se han centrado en la narrativa contemporánea y sobre todo en la obra de Carmen Martín Gaite.

Maria Vittoria Calvi is Professor of Spanish at the University of Milan. She has wide experience in research and higher education teaching. She is Vice-President of the Association of Spanish Scholars in Italy (for the period 2010-2014) 
and a member of CERLIS (Centro di Ricerca sui Linguaggi Specialistici) at the University of Bergamo. She has authored a great number of studies on Spanish Linguistics, focusing mainly on contrastive linguistics between Spanish and Italian and teaching-learning of related languages. Among her most recent research interests, contact between Spanish and Italian in migration contexts should be highlighted. In the literary field, her research mainly addresses contemporary fiction and, especially, the works of Carmen Martín Gaite. 